\title{
ANÁLISE DA LISTA VERMELHA DE ESPÉCIES DE AVES DA CAATINGA, BRASIL
}

Diego Fernandes Miranda a , Jean Nascimento dos Anjos ${ }^{\mathrm{b}}$.

a Pós-Graduando do Programa de Pós-Graduação em Ecologia: Teoria, Aplicação e Valores -UFBA. Rua Barão de Jeremoabo, 668, Campus de Ondina, Salvador-BA, Brasil. CEP: 40170-115.

b Pós-Graduando do Programa de Pós-Graduação em Ecologia: Teoria, Aplicação e Valores -UFBA. Rua Barão de Jeremoabo, 668, Campus de Ondina, Salvador-BA, Brasil. CEP: 40170-115.

\section{RESUMO}

A Caatinga tem sido apontada como uma importante área de endemismo para as aves sul-americanas, porém, a distribuição, a evolução e a ecologia da avifauna da região continuam pouco investigadas, refletindo, consequentemente, na política e ações de conservação. Este estudo objetivou listar as espécies de aves ameaçadas de extinção da Caatinga, bem como modelar a distribuição espacial dos táxons ameaçados de extinção. A lista de espécies de aves ocorrentes na Caatinga foi obtida com base na literatura específica e a busca pelas espécies ameaçadas foi realizada junto ao Livro Vermelho da fauna brasileira ameaçada de extinção do ICMBio. Os modelos cumulativos de riqueza de espécies (CSRM) foram feitos somando os resultados dos modelos binários previamente calculados. Avaliamos o estado de conservação de 548 espécies de aves ocorrentes na Caatinga e constatamos que 36 espécies se encontram ameaçadas de extinção. Conhecemos que parte das áreas de maior concentração de espécies ameaçadas encontra-se legalmente protegida. No entanto, ressaltamos que para melhorar a garantia de continuidade dessas espécies, é necessário a ampliação de áreas legalmente protegidas dentro do Bioma.

Palavras-chave: Avifauna, Caatinga, Distribuição espacial

\section{ABSTRACT}

The Caatinga has been identified as an important area of endemism for South American birds, however, the distribution, evolution and ecology of avifauna in the region remains poorly investigated, consequently reflecting on conservation policy and actions. This study aimed to list the endangered species of birds in the Caatinga, as well as to model the spatial distribution of endangered taxa. The list of bird species occurring in the Caatinga was obtained based on specific literature and the search for endangered species was carried out in the ICMBio's Red Book of endangered Brazilian fauna. The cumulative species richness models (CSRM) were made by adding the results of the previously calculated binary models. We assessed the conservation status of 548 bird species occurring in the Caatinga and found that 36 species are threatened with extinction. We know that part of the areas with the greatest concentration of threatened species is legally protected. However, we emphasize that to improve the guarantee of the continuity of these species, it is necessary to expand legally protected areas within the Biome.

Keywords: Avifauna, Caatinga, Spatial distribution

*Autor correspondente: Jean Nascimento dos Anjos. MSc em Ecologia: Teoria, Aplicação e Valores endereço completo. Tel: (71) 99183-575; E-mail: jeanjos2015@gmail.com 


\section{INTRODUÇÃO}

As listas vermelhas são consideradas uma ferramenta essencial para a conservação, fornecendo informações sobre as espécies ameaçadas, e desta forma permitindo que setores do governo, iniciativa privada e a sociedade priorizem ações em prol da conservação, minimizando os impactos sobre as espécies ameaçadas de extinção (DONALDSON, 2013). O Brasil teve a sua primeira lista de espécies ameaçadas elaborada em 1968, contando com 57 espécies em risco (ICMBio, 2018). Atualmente no Brasil, cerca de 1200 espécies se encontram em algum grau de ameaça, incluindo 236 espécies de aves $(11,9 \%$ das espécies avaliadas no Brasil) (ICMBio, 2018) e estas espécies sofrem impactos severos relacionados principalmente à perda de habitat e outros impactos humanos (ALVES et al. 2013).

A distribuição geográfica das espécies é limitada por uma série de fatores históricos, ecológicos e evolutivos, tais como as características climático-ambientais onde as espécies ocorrem, interações intra e interespecíficas, barreiras geográficas à dispersão, e a capacidade evolutiva das espécies de se adaptarem a novas condições climáticas (SOBERÓN; PETERSON, 2005). Diversos fatores ambientais, ecológicos e evolutivos, fazem com que as espécies não se distribuam de maneira cosmopolita no espaço geográfico, algumas sendo consideradas endêmicas, pois ocorrem, de maneira natural apenas em uma área geográfica restrita (PRIMACK, 2006). Áreas de endemismo, especialmente aquelas com alto grau de endemismo, devem ser priorizadas em políticas conservacionistas, pois espécies endêmicas geralmente ocupam nichos/habitats especializados e podem formar populações pequenas (THOMAS et al., 2004; PRIMACK, 2006; SILVA, 2017)

A Caatinga tem sido apontada como uma importante área de endemismo para as aves sul-americanas. Existem 548 espécies de aves pertencentes a 74 famílias neste bioma, que habitam um complexo gradiente ambiental criado por diversos tipos de florestas tropicais secas sazonais (ARAUJO; SILVA, 2017), porém, a distribuição, evolução e ecologia da avifauna desta região continuam pouco investigadas, refletindo consequentemente nas políticas e ações de conservação. A análise da composição da avifauna de uma localidade pode oferecer dados importantes sobre as características e o grau de conservação de um ambiente, além de que o conhecimento sobre as distribuições geográficas das espécies ajuda a definir planos sistemáticos para a conservação dos táxons e a priorização ou a criação de novas áreas protegidas (FAABORG, 1993; SILVA, 2017).

O Plano de Ação Nacional (PAN) para Conservação das Aves da Caatinga estabelece, dentre suas ações, identificar áreas prioritárias para conservação das espécies alvo do plano, com base em seus registros de ocorrência, além de recomendar a realização de expedições de busca que contemplem áreas de lacuna de conhecimento (ICMBio, 2014; MENDES et al., 2016). Portanto, conhecer quais e como estão distribuídas espacialmente as espécies de aves ameaçadas de extinção da Caatinga é importante, entre outras coisas, para melhor gerenciar estratégias de conservação visando garantir a continuidade dessas espécies na região. Assim, objetivamos neste estudo (1) listar as espécies de aves ameaçadas de extinção da Caatinga e, (2) modelar a distribuição espacial dos táxons ameaçados.

\section{MATERIAL E MÉTODOS}

\subsection{Espécies ameaçadas e registros de ocorrências}

A lista de espécies de aves ocorrentes na Caatinga foi obtida com base em Araujo \& Silva (2017). A partir desta lista, uma busca pelas espécies ameaçadas foi realizada junto ao Livro Vermelho da fauna brasileira ameaçada de extinção (ICMBio, 2018) onde até o momento da escrita deste artigo apresentava-se como a edição mais recente.

Para estimar a distribuição das aves da Caatinga, compilamos os registros de ocorrência de cada espécie ameaçada a partir do GBIF (Global Biodiversity Information Facility). 
Figura 1. Distribuição espacial dos registros do banco de dados das aves ameaçadas de extinção da Caatinga. Um total de 23.270 pontos de registros de 38 espécies ameaçadas foram inicialmente aplicados.

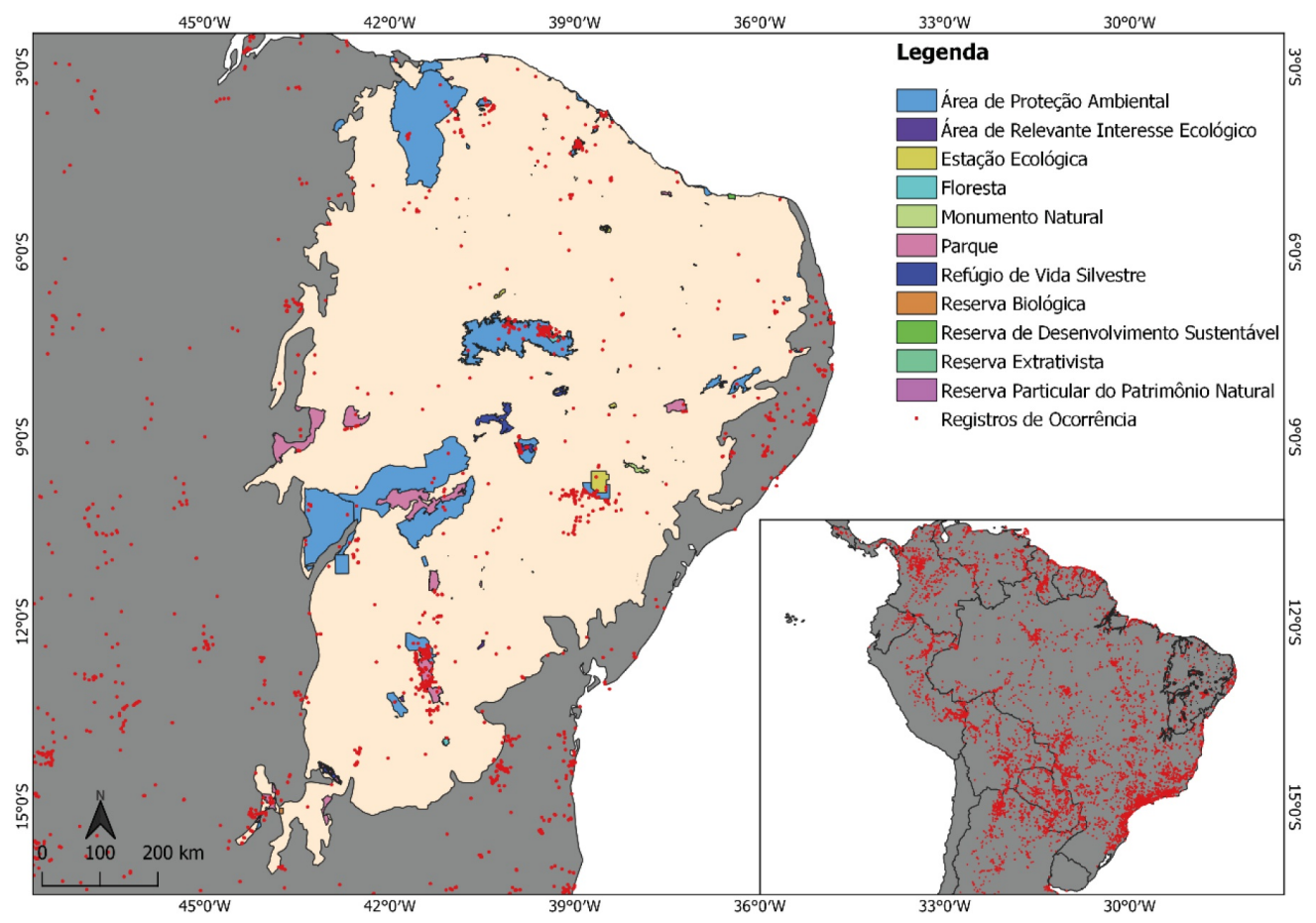

\subsection{Dados ambientais}

Variáveis climáticas para cenários atuais foram retiradas do banco de dados WorldClim (FICK; HIJMANS, 2017). Foram obtidas 19 variáveis bioclimáticas com uma resolução espacial de 30 segundos $\left(\sim 1 \mathrm{~km}^{2}\right)$. Para reduzir a autocorrelação entre as variáveis, uma análise de inflação da variância foi realizada, reduzindo para 10 variáveis analisadas (Tabela 1).

Tabela 1. Variáveis ambientais disponíveis para o Bioma Caatinga

\begin{tabular}{|c|c|c|c|c|}
\hline N. & Variável & $\begin{array}{c}\text { Tipo de da- } \\
\text { dos }\end{array}$ & Resolução & Fonte \\
\hline 1 & Isotermabilidade & Contínuo & $1 \mathrm{~km}^{2}$ & URL: http://worldclim.org \\
\hline 2 & Sazonalidade de temperatura & Contínuo & $1 \mathrm{~km}^{2}$ & URL: http://worldclim.org \\
\hline 3 & Temperatura máxima do mês mais quente & Contínuo & $1 \mathrm{~km}^{2}$ & URL: http://worldclim.org \\
\hline 4 & Temperatura mínima do mês mais frio & Contínuo & $1 \mathrm{~km}^{2}$ & URL: http://worldclim.org \\
\hline 5 & Temperatura média do trimestre mais úmido & Contínuo & $1 \mathrm{~km}^{2}$ & URL: http://worldclim.org \\
\hline 6 & Precipitação anual & Contínuo & $1 \mathrm{~km}^{2}$ & URL: http://worldclim.org \\
\hline 7 & Precipitação do mês mais seco & Contínuo & $1 \mathrm{~km}^{2}$ & URL: http://worldclim.org \\
\hline 8 & Sazonalidade de precipitação & Contínuo & $1 \mathrm{~km}^{2}$ & URL: http://worldclim.org \\
\hline 9 & Precipitação do trimestre mais quente & Contínuo & $1 \mathrm{~km}^{2}$ & URL: http://worldclim.org \\
\hline 10 & Precipitação do trimestre mais frio & Contínuo & $1 \mathrm{~km}^{2}$ & URL: http://worldclim.org \\
\hline 11 & Temperatura média anual & Contínuo & $1 \mathrm{~km}^{2}$ & URL: http://worldclim.org \\
\hline 12 & Faixa diurna média & Contínuo & $1 \mathrm{~km}^{2}$ & URL: http://worldclim.org \\
\hline 13 & Faixa anual de temperatura & Contínuo & $1 \mathrm{~km}^{2}$ & URL: http://worldclim.org \\
\hline 14 & Temperatura média do trimestre mais seco & Contínuo & $1 \mathrm{~km}^{2}$ & URL: http://worldclim.org \\
\hline 15 & Temperatura média do trimestre mais quente & Contínuo & $1 \mathrm{~km}^{2}$ & URL: http://worldclim.org \\
\hline 16 & Temperatura média do trimestre mais frio & Contínuo & $1 \mathrm{~km}^{2}$ & URL: http://worldclim.org \\
\hline 17 & Precipitação do mês mais chuvoso & Contínuo & $1 \mathrm{~km}^{2}$ & URL: http://worldclim.org \\
\hline 18 & Precipitação do trimestre mais úmido & Contínuo & $1 \mathrm{~km}^{2}$ & URL: http://worldclim.org \\
\hline 19 & Precipitação do quarto mais seco & Contínuo & $1 \mathrm{~km}^{2}$ & URL: http://worldclim.org \\
\hline
\end{tabular}

*As primeiras dez variáveis foram selecionadas para os modelos de nicho. 


\subsection{Análise dos dados e modelagem ecológica}

Os dados de ocorrência obtidos a partir do GBIF foram filtrados com o intuito de remover possíveis vieses de levantamento (efeito museu) (PHILLIPS, 2009). Para isso, foram utilizados buffers circulares com $25 \mathrm{~km}$ de raio através do software $R$ ( $R$ Core Team, 2019), deixando apenas um ponto de ocorrência de cada espécie dentro destas áreas. Espécies com menos de 8 pontos de ocorrência foram removidas da análise de acordo com os critérios de Tognelli et al (2011). Assim, um total de 5.110 pontos de ocorrência foram utilizados para a modelagem de distribuição potencial.

A distribuição potencial de cada espécie foi calculada com base no modelo de Máxima Entropia (MaxEnt; PHILLIPS et al. 2006). Foram realizadas 100 replicações para cada espécie em um conjunto de algoritmos de subamostragem onde os dados de ocorrência de cada espécie foram divididos em grupos de calibração (70\%) e avaliação (30\%). O desempenho dos modelos foi verificado a partir da análise da curva ROC (Receiver Operating Characteristic) (YUAN et al., 2015) e do máximo TSS (True Skill Statistics) (ALLOUCHE, et al., 2006). A área abaixo da curva ROC (Area Under the Curve - AUC) foi utilizada como estimativa de desempenho do modelo seguindo o proposto por Pearce \& Ferrier (2000), onde predições abaixo de 0.75 foram descartadas (ELITH; BURGMAN, 2002). Já o TSS foi utilizado para uma avaliação com maior acurácia e precisão dos modelos após a análise do AUC, onde as predições abaixo de 0.5 foram descartadas (PRÉAU et al., 2018).

\subsection{Modelo de riqueza cumulativa de espécies}

Criamos modelos cumulativos de riqueza de espécies (CSRM) somando os resultados dos modelos binários obtidos (ORTEGAANDRADE et al., 2021), onde as espécies foram classificadas como presentes $(1=$ se 0 pixel corresponde a uma área com potencial para ocorrência da espécie acima de 0.5) ou ausente $(0=$ se 0 potencial de ocorrência no pixel for abaixo de 0.5).

\section{RESULTADOS E DISCUSSÃO}

Avaliamos o estado de conservação de 548 espécies de aves ocorrentes na Caatinga. Desse total, 35 espécies estão em categorias de ameaças de extinção. A maior parte dos táxons ameaçados (67\%) ocorre em categorias de maior risco de extinção (Crítico - CR, Em Perigo - EN ou Vulnerável - VU). Por outro lado, 33\% figuram na categoria Quase Ameaçada (NT) (Tabela 2). Araujo \& Silva (2017) apontam que, dentre as espécies ameaçadas de extinção, cerca de $80 \%$ apresentam baixa capacidade adaptativa, ou seja, podem apenas ser encontradas em paisagens compostas por ecossistemas intactos ou quase intactos. Isto é especialmente preocupante uma vez que mais de $60 \%$ do bioma é composto por ecossistemas antrópicos (DA SILVA; BARBOSA, 2017) e a intervenção humana, incluindo queimadas e desmatamentos, aceleram a degradação do potencial florestal e dos solos do bioma, provocando intensos desequilíbrios ecológicos (ALVES et al. 2009). Além disso, cerca de $44 \%$ das espécies ameaçadas são endêmicas do bioma (PACHECO, 2004) (Tabela 2). De acordo com Garda et al. (2018) alguns desses endemismos são associados à vegetação mais arbustiva e aberta, mas outros ocorrem estritamente em ambientes florestais, incluindo enclaves de florestas úmidas. Assim, torna-se evidente, portanto, a importância da conservação dos habitats naturais remanescentes da Caatinga, incluindo a maior gama de fitofisionomias possível, a fim de melhor assegurar a continuidade dessas espécies na região. 
Tabela 2. Espécies de aves em categorias de risco de extinção com seus registros de ocorrência, AUC e TSS usados na criação do modelo para cada espécie ameaçada de extinção avaliada neste estudo.

\begin{tabular}{|c|c|c|c|c|c|}
\hline Nome científico & $\begin{array}{l}\text { Registros de } \\
\text { ocorrência }\end{array}$ & AUC & TSS & $\begin{array}{c}\text { Risco (ICM- } \\
\text { Bio) }\end{array}$ & $\begin{array}{c}\text { Endêmica (Pacheco, } \\
2004)\end{array}$ \\
\hline Amazona aestiva & 1029 & 0.856 & 0.598 & NT & NÃO \\
\hline Amazona vinacea & 134 & 0.961 & 0.864 & vu & NÃO \\
\hline Anodorhynchus leari & 13 & 0.804 & 0.7 & EN & SIM \\
\hline Antilophia bokermanni & 5 & NA & NA & $\mathrm{CR}$ & SIM \\
\hline Ara chioropterus & 1006 & 0.779 & 0.482 & NT & NÃO \\
\hline Arremon franciscanus & 10 & 0.968 & 0.943 & NT & SIM \\
\hline Augastes lumachella & 18 & 0.884 & 0.803 & EN & SIM \\
\hline Buteogallus aequinoctialis & 67 & 0.954 & 0.837 & NT & NÃO \\
\hline Conopophaga cearae & 27 & 0.981 & 0.929 & EN & NÃO \\
\hline Crypturellus noctivagus & 96 & 0.939 & 0.814 & $v^{x}$ & $\mathrm{SIM}^{x}$ \\
\hline Curaeus forbesi & 13 & 0.908 & 0.857 & vu & NÃO \\
\hline Cyanopsitta spixï & 1 & NA & NA & $\mathrm{CR}$ & SIM \\
\hline Euscarthmus rufomarginatus & 53 & 0.779 & 0.555 & NT & NÃO \\
\hline Formicivora grantsaui & 10 & 0.776 & 0.73 & EN & SIM \\
\hline Formicivora iheringi & 21 & 0.806 & 0.657 & NT & SIM \\
\hline Harpyhaliaetus coronatus & 321 & 0.894 & 0.683 & EN & NÃO \\
\hline Hemitriccus mirandae & 17 & 0.969 & 0.913 & vu & SIM \\
\hline Hyiopezus ochroleucus & 61 & 0.939 & 0.849 & NT & SIM \\
\hline Knipolegus franciscanus & 18 & 0.93 & 0.92 & NT & NÃO \\
\hline Lepidocolaptes wagleri & 4 & NA & NA & EN & NÃO \\
\hline Neomorphus geoffroyi & 74 & 0.905 & 0.723 & vu & NÃO \\
\hline Nyctiprogne vielliardi & 17 & 0.985 & 0.976 & NT & NÃO \\
\hline Penelope jacucaca & 44 & 0.8 & 0.771 & vu & SIM \\
\hline Phylloscartes beckeri & 12 & 0.846 & 0.792 & EN & NÃO \\
\hline Phylloscartes roquettei & 22 & 0.891 & 0.824 & EN & SIM \\
\hline Primolius maracana & 362 & 0.876 & 0.622 & NT & SIM \\
\hline Procnias nudicollis & 212 & 0.971 & 0.888 & NT & NÃO \\
\hline Pymhura cruentata & 38 & 0.888 & 0.82 & vu & NÃO \\
\hline Pymhura griseipectus & 10 & 0.734 & 0.59 & EN & NÃO \\
\hline Rhopornis ardesiacus & 12 & 0.859 & 0.812 & EN & SIM \\
\hline Sarcoramphus papa & 1270 & 0.711 & 0.349 & NT & NÃO \\
\hline Sclerurus cearensis & 2 & NA & NA & vu & NÃO \\
\hline Scytalopus diamantinensis & 8 & 0.872 & 0.85 & EN & SIM \\
\hline Spinus yarrellii & 7 & NA & NA & vu & NÃO \\
\hline Taoniscus nanus & 16 & 0.885 & 0.828 & EN & NÃO \\
\hline Xiphocolaptes falcirostris & 32 & 0.873 & 0.722 & vu & SIM \\
\hline
\end{tabular}

*Subespécie Crypturellus noctivagus zabele.

A modelagem de espécies ameaçadas (CSRM) demonstrou um valor máximo de 24 espécies sobrepostas por pixel (figura 2). A maior concentração de táxons ameaçados ocorre na porção Sul do Bioma Caatinga (interior do Estado da Bahia) região que detém cerca de $11 \%$ das Unidades de Conservação existentes no bioma Caatinga (figura 1). Entretanto, grande parte da área de maior concentração de espécies ameaçadas, está fora dos limites de UCs e são constantemente pressionadas por atividades humanas, 
pincipalmente, atividades ligadas a perda ou modificação do habitat (agricultura, pecuária e crescimento urbano). De acordo com Pereira et al. (2010) a perda de habitats naturais é apontada como a causa primordial do declínio da biodiversidade em diferentes ecossistemas ao longo do mundo, especialmente áreas com alto endemismo e com baixa cobertura de proteção legal a exemplo da Caatinga. Segundo Prestes et al. (2018) apenas 8,87\% do território total da Caatinga $(828.000 \mathrm{Km} 2)$ é protegido em Unidades de Conservação. A modificação do habitat, entre outros efeitos, pode comprometer os recursos utilizados por assembleias de aves especialistas, chegando a alterar padrões de distribuição de espécies que não são adaptadas às novas condições (ALEIXO, 1999) e por consequência, alterar profundamente a composição e funcionalidade da assembleia de aves da região.

Figura 2. Riqueza cumulativa de espécies ameaçadas de extinção por categoria de ameaça da Lista Vermelha do ICMBio (2018). O mapa para táxons da categoria CR não foi gerado por conter apenas duas espécies que não foram modeladas: Antilophia bokermanni e Cyanopsitta spix

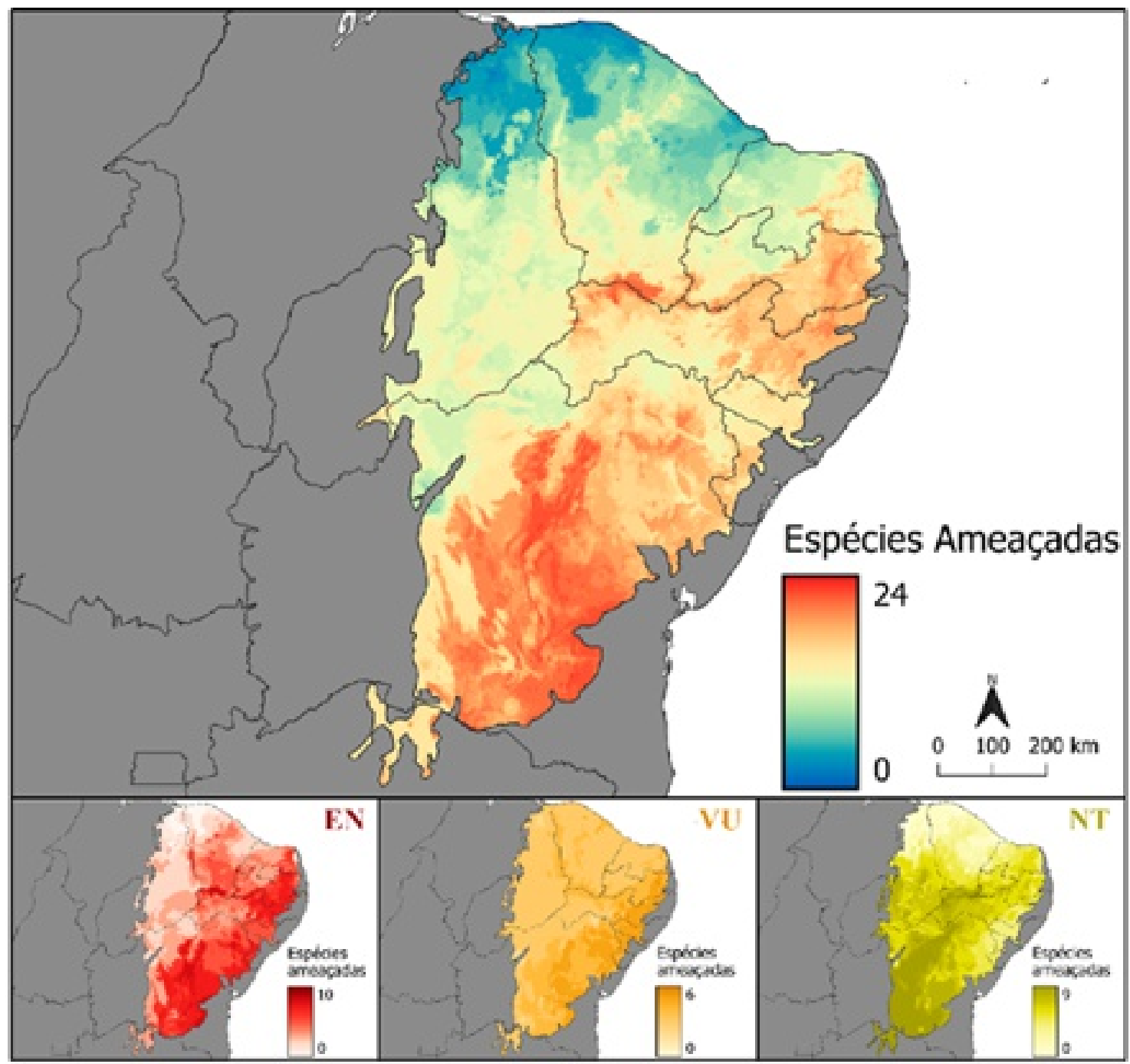

Uma outra área de grande concentração de táxons ameaçados estende-se entre os Estados de Pernambuco e Ceará. Esta área está totalmente inserida nas APAs Chapada do Araripe e Floresta Nacional do Araripe-Apodi, que são UCs federais de uso sustentável, essenciais para manutenção da fauna local, sobretudo para continuidade de espécies ameaçadas extinção, tendo em vista suas importâncias para proteção legal de áreas com alta biodiversidade. Contudo, de acordo com Silva (2017) apenas $70.000 \mathrm{~km}^{2}$ (cerca de 1\%) do território da Caatinga encontra-se inserido em unidades de conservação integrais. Comparando este valor com o de outros biomas brasileiros, este número aproxima-se 
ao valor de UCs do Pampa (0,8\%), mas é bem menor que na Amazônia (49\%), no Cerrado (7.7\%), no Pantanal (3,9\%), e na Mata Atlântica (3,2\%) (OLIVEIRA et al., 2017; SILVA, 2017). Dessa forma, ressaltamos que a ampliação, bem como a criação de novas UCs, em especial, de uso permanente em áreas sensíveis a perda de espécies ameaçadas na Caatinga, poderia garantir maior proteção e, portanto, maior seguro de continuidade para os táxons ameaçados frente aos impactos antrópicos ocorrentes no Bioma.

\section{CONCLUSÃO}

A avifauna do Bioma Caatinga, apresenta um número expressivo de espécies sob ameaça de extinção. Constatamos que a maioria dos táxons com algum grau de ameaça, encontra-se na categoria de risco crítico de ameaça de extinção e pouco menos da metade dessas espécies são endêmicas da Caatinga.

Constatamos que uma parte importante das áreas de maior concentração de espécies ameaçadas encontra-se protegida legalmente. Entretanto, ressaltamos que para garantir maior seguro de continuidade das espécies ameaçadas dentro da Caatinga, é imprescindível a ampliação de áreas legalmente protegidas dentro do Bioma.

Ressaltamos, portanto, a necessidade de estudos mais abrangentes sobre a distribuição das espécies da Caatinga, incluindo táxons animais e vegetais a fim de determinar com maior precisão a existência de áreas sensíveis a perda de espécies dentro do Bioma.

\section{CONFLITOS DE INTERESSE}

Não há conflito de interesse na pesquisa.

\section{REFERÊNCIAS}

ALEIXO, A., 1999. Effects of selective logging on a bird community in the Brazilian Atlantic Forest. The Condor, 101(3), pp.537548.

ALLOUCHE, $O$. et al. Assessing the accuracy of species distribution models: prevalence, kappa and the true skill statistic (TSS). Journal of Applied Ecology, v. 43, n. 6, 2006.

ALVES, J.J.A., DE ARAÚJO, M.A. AND DO NASCIMENTO, S.S., 2009. Degradação da Caatinga: uma investigação ecogeográfica. Revista Caatinga, 22(3), pp.126-135.

ALVES, RÔMULO ROMEU NÓBREGA et al. Ethno-ornithology and conservation of wild birds in the semi-arid Caatinga of northeastern Brazil. Journal of Ethnobiology and Ethnomedicine, v. 9, n. 1, p. 1-12, 2013.

ARAUJO, HELDER FARIAS PEREIRA; DA SILVA, JOSÉ MARIA CARDOSO. The avifauna of the Caatinga: biogeography, ecology, and conservation. In: Caatinga. Springer, Cham, 2017. p. 181-210.

DA SILVA, J.M.C. AND BARBOSA, L.C.F., 2017. Impact of human activities on the Caatinga. In Caatinga (pp. 359-368). Springer, Cham.

DOBSON, ANDREW et al. Habitat loss, trophic collapse, and the decline of ecosystem services. Ecology, v. 87, n. 8, p. 1915-1924, 2006.

DONALDSON, J. O livro vermelho da flora do Brasil - enfrentando um desafio. In: Livro vermelho da Flora do Brasil. 2013. Orgs.: Martinelli, G. \& Moraes, M.A. 1. ed. - Rio de Janeiro, Instituto de Pesquisas Jardim Botânico do Rio de Janeiro. p. 9.

ELITH, J. AND BURGMAN, M.A., 2002. Predictions and their validation: rare plants in the Central Highlands, Victoria, Australia. Predicting species occurrences: issues of accuracy and scale, pp.303-314.

GARDA, ADRIAN ANTONIO et al. Os animais vertebrados do Bioma Caatinga. Ciência e Cultura, v. 70, n. 4, p. 29-34, 2018. 
fragmentation in the temperate zone: a perspective for managers. Status and Management of Neotropical Migratory Birds: September 21-25, 1992, Estes Park Center, YMCA of the Rockies, Colorado, 229, p.331.

FICK, STEPHEN E.; HIJMANS, ROBERT J. WorldClim 2: new 1-km spatial resolution climate surfaces for global land areas. International journal of climatology, v. 37, n. 12, p. 4302-4315, 2017.

ICMBio. Aprova o Plano de Ação Nacional para Conservação das Aves Ameaçadas da Caatinga. Portaria $n^{\circ}$ 92, de 02 de setembro de 2014. . 2014.

ICMBio. Livro Vermelho da Fauna Brasileira Ameaçada de Extinção: Volume 1 / -1. ed. -- Brasília, DF: ICMBio/MMA, 2018.

MENDES, D.; EMANUEL, A.; ALVES DE SOUSA, B. Avifauna de uma área de Caatinga arbórea e outros ambientes na região do Sertão paraibano, Brasil.Ornithologia. [s.l: s.n.]. Disponível em: <http://geocatalogo.mma.gov.br/ mma>. Acesso em: 6 jun. 2021.

ORTEGA-ANDRADE, H. MAURICIO et al. Red List assessment of amphibian species of Ecuador: A multidimensional approach for their conservation. PloS one, v. 16, n. 5, p. e0251027, 2021.

PACHECO, J.F., 2004. As aves da Caatinga: uma análise histórica do conhecimento. Biodiversidade da Caatinga: áreas e ações prioritárias para a conservação, pp.189-250.

PEARCE, J. AND FERRIER, S., 2000. An evaluation of alternative algorithms for fitting species distribution models using logistic regression. Ecological modelling, 128(2-3), pp.127-147.

PEREIRA, HENRIQUE $M$. et al. Scenarios for global biodiversity in the 21st century. Science, v. 330 , n. 6010 , p. 14961501, 2010.
PHILLIPS, S.J., ANDERSON, R.P. AND SCHAPIRE, R.E., 2006. Maximum entropy modeling of species geographic distributions. Ecological modelling, 190(3-4), pp.231-259.

PHILLIPS, STEVEN J. et al. Sample selection bias and presence-only distribution models: implications for background and pseudo-absence data. Ecological applications, v. 19, n. 1, p. 181-197, 2009.

PREAU, Clementine et al. Modeling potential distributions of three European amphibian species comparing ENFA and Maxent. Herpetological Conservation and Biology, v. 13, n. 1, p. 91-104, 2018.

PRESTES, LAURA DIAS; PERELLO, LUÍS FERNANDO CARVALHO; GRUBER, NELSON LUIS SAMBAQUI. Métodos para avaliar efetividade de gestão: o caso particular das Áreas de Proteção Ambiental (APAs). Desenvolvimento e Meio Ambiente, v. 44, 2018.

PRIMACK, R. B. Essentials of Conservation Biology. Sunderland, Mass: Sinauer Associates, 2006.

$R$ CORE TEAM. R: A language and environment for statistical computing. $R$ Foundation for Statistical Computing, Vienna, Austria. URL https://www.R-project.org/, 2009.

SILVA, Marcos Vinicios Alexandre da. Modelagem de distribuição geográfica das aves endêmicas da Caatinga, status de conservação e possíveis efeitos de mudanças climático-ambientais. 2017. Dissertação de Mestrado. Universidade Federal de Pernambuco.

SOBERÓN, JORGE; PETERSON, A. TOWNSEND. Interpretation of models of fundamental ecological niches and species' distributional areas. 2005.

THOMAS, JEREMY A. et al. Comparative losses of British butterflies, birds, and plants and the global extinction crisis. Science, v. 303, 
n. 5665, p. 1879-1881, 2004.

TOGNELLI, M.F., ABBA, A.M., BENDER, J.B. AND SEITZ, V.P., 2011. Assessing conservation priorities of xenarthrans in Argentina. Biodiversity and Conservation, 20(1), pp.141-151.

YUAN, H.S., WEI, Y.L. AND WANG, X.G., 2015. Maxent modeling for predicting the potential distribution of Sanghuang, an important group of medicinal fungi in China.

Fungal Ecology, 17, pp.140-145. 